\title{
INTERACTION DE FLUIDES POTENTIELS AVEC UNE MEMBRANE ÉLASTIQUE
}

\author{
Yvon MADAY ${ }^{1,2}$, Bertrand MAURY ${ }^{1}$ and Paul METIER ${ }^{1}$
}

\begin{abstract}
We deal with an unsteady bidimensionnal fluid-structure interaction problem. A rigid cavity is filled with two incompressible inviscid homogeneous fluids separated by an elastic wall undergoing small transversal displacements. The fluids are incompressible and curl-free; from that point, we study potential fluids in moving domains. The coupling is done in two ways: continuity of the normal velocities and action-reaction principle at the interface.

After an energy estimates study to valid the model, we present a simulation using ALE approach for the fluids and spectral method to discretize the membrane transversal displacement.
\end{abstract}

Résumé. Nous considérons un problème d'interaction fluide-structure instationnaire dans un domaine bidimensionnel. Le bord de la cavité, remplie par les fluides, est formé d'une partie rigide et d'une partie élastique sur laquelle on considère une équation d'ondes (pour modéliser de petits déplacements transversaux de la membrane). Les fluides sont supposés parfaits, incompressibles, homogènes et irrotationnels. Les conditions de transmissions traduisent la continuité des vitesses normales et le principe d'action-réaction à l'interface.

Après l'obtention des estimations d'énergie qui valident en un sens le modèle, nous présentons une simulation utilisant des formulations ALE pour les fluides, et une méthode spectrale pour le déplacement membranaire transverse.

AMS Subject Classification. 65M06, 65M70, 65C20, 76B10 .

The dates will be set by the publisher.

\section{ModÉLISATION}

Nous nous intéressons à la modélisation bidimensionnelle d'un ventricule d'un coeur artificiel. Ce ventricule est constitué de deux poches rigides, chacune remplie par un fluide, et séparées par une paroi élastique. La première poche, remplie par un fluide assimilable à une huile, possède une entrée qui permet une injection de fluide, et une sortie libre. La seconde cavité, remplie de sang, possède un système d'entrée-sortie libres: une entrée par laquelle le sang veineux non-oxygéné (respectivement le sang oxygéné) pénètre dans la cavité du ventricule droit (respectivement gauche), et une sortie qui assure la circulation du sang vers l'artère pulmonaire (respectivement qui alimente l'aorte en sang oxygéné). Ces portes ne sont par ailleurs jamais ouvertes en même temps.

\footnotetext{
Keywords and phrases: Potential fluid, Linear elasticity, Fluid-Structure Interaction, Moving domain, ALE approach.

1 Laboratoire d'Analyse Numérique, 175,rue du Chevaleret 75013 PARIS ; e-mail: maday@ann.jussieu.fr,

e-mail: maury@ann.jussieu.fr, e-mail: metier@ann.jussieu.fr.

2 Laboratoire ASCI, Bât. 506, Université Paris Sud, 91405 Orsay, France.
}

(c) EDP Sciences, SMAI 2001 


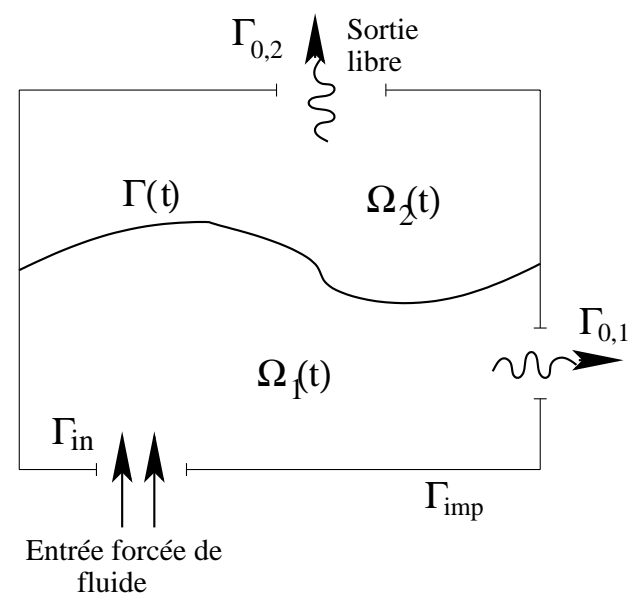

Figure 1. Cavité remplie par deux fluides potentiels séparés par une membrane élastique.

Pour modéliser ce système en première approximation, nous considérons une cavité bidimensionnelle rigide remplie par deux fluides potentiels homogènes séparés par une membrane élastique (voir Figure 1). Ainsi le caractère non newtonien du sang, et la viscosité du fluide assimilé à une huile ne sont pas pris en compte. Le problème reste néanmoins instationnaire et non-linéaire (dû à la mobilité des domaines fluides et structure). Par ailleurs, dans la seconde cavité, l'alternance de l'ouverture d'une des deux portes, ainsi que la modélisation par une même condition au bord, permettent de ne considérer physiquement qu'une seule porte. Enfin, seuls les déplacements transverses de la membrane sont modélisés.

\subsection{Structure}

Pour la structure monodimensionnelle, le déplacement transverse $d$ (composante du déplacement selon $\mathbf{e}_{y}$ pour fixer les idées) est modélisé par une équation du type visco-élastique, ce qui se traduit en coordonnées lagrangiennes par le système suivant sur l'intervalle $\gamma=] 0, L[$ :

$$
\left\{\begin{aligned}
\rho_{s} \partial_{t}^{2} d-\sigma \partial_{x}^{2} d-K \partial_{x}^{2} \partial_{t} d & =\left(\mathrm{T}_{\mathrm{FL}} \mathbf{n}_{0}\right)_{y} & & \text { dans } \gamma \times] 0, T[ \\
d(x, t) & =0 & & \text { sur } \partial \gamma \times] 0, T[ \\
{\left[d(x, 0), \partial_{t} d(x, 0)\right] } & =\left[d_{0}, d_{1}\right] & & \text { dans } \gamma
\end{aligned}\right.
$$

où $\partial_{t}$ (resp. $\partial_{x}$ ) désigne l'opérateur de la dérivée partielle en temps (resp. selon la variable d'espace $x \in \gamma$ ); $\left(\rho_{s}, \sigma, K\right)$ est le triplet de constantes caractéristique de la membrane; $\left(\mathrm{T}_{\mathrm{FL}} \mathbf{n}_{0}\right)_{y}$ est la seconde composante du tenseur des contraintes fluides appliqué à la normale à la paroi $\gamma$, et réécrit en coordonnées lagrangiennes; $d_{0}$ et $d_{1}$ sont respectivement la position et la vitesse initiales données. Pour $t \in[0, T]$, nous posons:

$$
\begin{aligned}
& \chi(t, \cdot): \quad \gamma \rightarrow \Gamma(t) \\
& \xi \mapsto(\xi, d(\xi, t))
\end{aligned}
$$

C'est une bijection qui permet de passer aisément des coordonnées lagrangiennes (sur $\gamma$ ) aux coordonnées eulériennes $(\operatorname{sur} \Gamma(t))$.

\subsection{Fluides}

Nous considérons d'autre part un fluide parfait incompressible homogène et irrotationnel dans chacune des deux cavités $\Omega_{1}(t)$ et $\Omega_{2}(t)$. Puisque notre étude se place dans un cadre bidimensionnel, la vitesse eulérienne 
$\mathbf{u}_{i}$ du fluide dans $\Omega_{i}(t)$, pour $i \in\{1,2\}$, s'écrit $\mathbf{u}_{i}=\nabla \phi_{i}$, où $\phi_{i}$ est une fonction à valeurs scalaires dépendant du temps et de l'espace. Les équations vérifiées par les deux potentiels sont les suivantes:

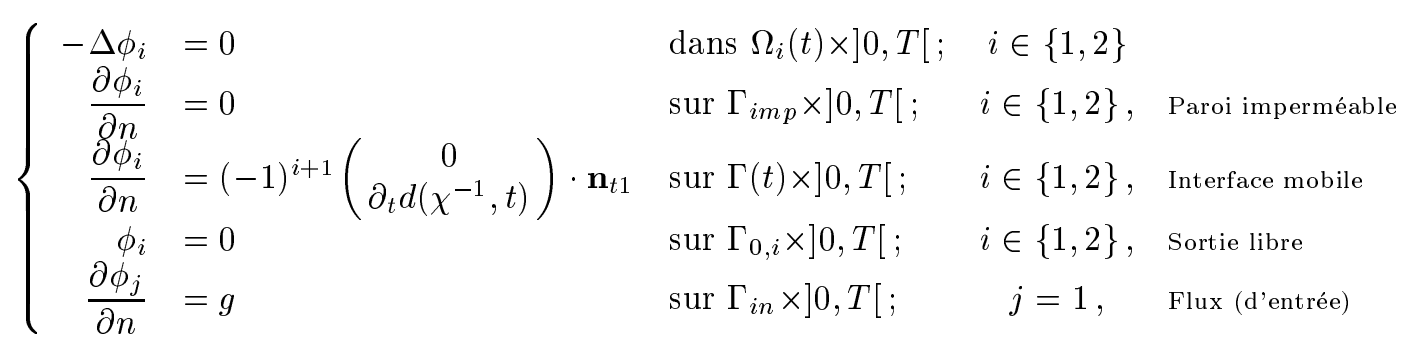

où $\mathbf{n}_{t 1}$ désigne la normale unitaire à la paroi mobile $\Gamma(t)$ extérieure à $\Omega_{1}(t)$ et dépendant du temps; $g$ est une fonction scalaire qui modélise une injection forcée de fluide dans $\Omega_{1}(t)$, et dont l'apport d'énergie au système sera abordé plus loin.

Remarque 1.1. Les problèmes vérifiés par les potentiels sont elliptiques, et aucun opérateur différentiel en temps n'agit sur $\phi_{i}$. La dépendance en temps de $\phi_{i}$ tient uniquement à trois points: le flux g, la condition limite sur l'interface, et évidemment la mobilité du domaine.

Enfin, on retrouve la pression (eulérienne) $P_{i}$ dans chacune des cavités grâce à la formule de Bernoulli pour un fluide parfait homogène incompressible irrotationnel:

$$
\frac{\partial \phi_{i}}{\partial t}+\frac{\left|\nabla \phi_{i}\right|^{2}}{2}+\frac{P_{i}}{\rho_{i}}=\frac{\beta_{i}(t)}{\rho_{i}}
$$

où $\rho_{i}$ est la masse volumique du fluide $i \in\{1,2\}$, et $\beta_{i}(t)$ désigne une fonction constante en espace qui ne dépend que du temps.

Remarque 1.2. La formule de Bernoulli est obtenue en intégrant l'équation d'Euler:

$$
\frac{\partial \mathbf{u}_{i}}{\partial t}+\mathbf{u}_{i} \cdot \nabla \mathbf{u}_{i}+\frac{\nabla P_{i}}{\rho_{i}}=0
$$

(régissant l'écoulement d'un fluide parfait), en réécrivant:

$$
\mathbf{u}_{i} \cdot \nabla \mathbf{u}_{i}=\nabla\left(\frac{\left|\mathbf{u}_{i}\right|^{2}}{2}\right)+\left(\operatorname{rot} \mathbf{u}_{i}\right) \wedge \mathbf{u}_{i}
$$

et en utilisant le caractère irrotationnel et l'écriture sous forme de potentiel $\mathbf{u}_{i}=\nabla \phi_{i}$. De làa, grâce à l'homogénéité du fluide, on obtient:

$$
0=\frac{\partial \mathbf{u}_{i}}{\partial t}+\mathbf{u}_{i} \cdot \nabla \mathbf{u}_{i}+\frac{\nabla P_{i}}{\rho_{i}}=\nabla\left(\frac{\partial \phi_{i}}{\partial t}+\frac{\left|\nabla \phi_{i}\right|^{2}}{2}+\frac{P_{i}}{\rho_{i}}\right) .
$$

Ainsi on a la formule suivante en chaque point $M$ du domaine fluide $\Omega_{i}(t)$ (simplement connexe):

$$
\frac{\partial \phi_{i}}{\partial t}(t, M)+\frac{\left|\nabla \phi_{i}(t, M)\right|^{2}}{2}+\frac{P_{i}(t, M)}{\rho_{i}}=\frac{\beta_{i}(t)}{\rho_{i}}
$$

où la fonction $\beta_{i}(t)$ est une constante en espace égale à:

$$
\beta_{i}(t)=\beta_{i}(t, N)=\rho_{i} \frac{\partial \phi_{i}}{\partial t}(t, N)+\rho_{i} \frac{\left|\nabla \phi_{i}(t, N)\right|^{2}}{2}+P_{i}(t, N)
$$


pour un point $N$ quelconque fixé dans la i-ème cavité. Elle est assimilable à une pression de référence pour une mise à l'échelle de la pression dans chaque cavité. Dans le cas présent, chaque cavité possède une sortie libre, et nous pouvons considérer que le fluide passant par la paroi $\Gamma_{0, i}$ rejoint un réservoir $\mathcal{R}_{i}$ annexe. Nous pouvons alors choisir $N$ situé dans $\mathcal{R}_{i}$. On peut même aller jusqu'à prendre $\beta_{i} \equiv 0$ pour $i=1$ et 2 , mais la liberté de ces paramètres est exploitable par la suite.

\subsection{Tenseur des contraintes}

Pour un fluide parfait (donc non visqueux), le tenseur des contraintes fluides est restreint à l'expression de la pression. Dans le cas présent, c'est la différence de pression qui apparaît, et son expression réécrite en coordonnées lagrangiennes est:

$$
\mathrm{T}_{\mathrm{FL}}=-\left(\left(\left[\beta_{1}-P_{1}\right]-\left[\beta_{2}-P_{2}\right]\right) \circ \chi\right) \mathrm{Id},
$$

où $\chi$ est la fonction introduite en (2) qui permet de passer de la position lagrangienne $\gamma$ de l'interface à sa position eulérienne $\Gamma(t)$, et Id est la matrice identité.

En notant $\Sigma_{S}$ le tenseur des contraintes de la structure, le principe d'action-réaction se traduit par l'égalité des contraintes normales à l'interface, c'est à dire:

$$
\Sigma_{S} \mathbf{n}_{0}=\mathrm{T}_{\mathrm{FL}} \mathbf{n}_{0}=-\left(\left(\left[\beta_{1}-P_{1}\right]-\left[\beta_{2}-P_{2}\right]\right) \circ \chi\right) \mathbf{n}_{0} .
$$

\subsection{Synthèse des éléments de couplage}

\begin{tabular}{|c|c|c|c|}
\hline $\mathbf{1}$ & $\begin{array}{c}\text { Coïncidence } \\
\text { des interfaces }\end{array}$ & $\begin{array}{c}\text { L'interface lagrangienne } \gamma \text { de référence et } \\
\text { l'interface eulérienne } \Gamma(t) \text { déformée } \\
\text { sont en bijection à chaque instant. }\end{array}$ & $\chi(t, \cdot): \gamma \leftrightarrow \Gamma(t)$ \\
\hline $\mathbf{2}$ & $\begin{array}{c}\text { Continuité des } \\
\text { vitesses normales } \\
\text { à l'interface }\end{array}$ & $\begin{array}{c}\text { C'est une condition de type Dirichlet sur la } \\
\text { vitesse } \mathbf{u} \text { qui se traduit sur les potentiels par } \\
\text { une condition de Neumann. }\end{array}$ & $\begin{array}{c}\nabla \phi_{1} \cdot \mathbf{n}_{t 1}=-\nabla \phi_{2} \cdot \mathbf{n}_{t 1} \\
=\left(\partial_{t} d \circ \chi^{-1}\right)\left(\mathbf{n}_{t 1}\right)_{y}\end{array}$ \\
\hline $\mathbf{3}$ & $\begin{array}{c}\text { Bilan des } \\
\text { contraintes à } \\
\text { l'interface }\end{array}$ & $\begin{array}{c}\text { Egalité du tenseur des contraintes normales } \\
\text { des fluides et de celui de la structure } \\
\text { à l'interface. }\end{array}$ & $\begin{array}{c}\Sigma_{S} \mathbf{n}_{0}=\mathrm{T}_{\mathrm{FL}} \mathbf{n}_{0}=\left(\left[P_{1}-P_{2}\right] \circ \chi\right. \\
\left.-\left[\beta_{1}-\beta_{2}\right]\right) \mathbf{n}_{0}\end{array}$ \\
\hline
\end{tabular}

\subsection{Autre modélisation possible}

Plutôt que de prendre $\frac{\partial \phi_{1}}{\partial n}=g$ sur $\Gamma_{i n}$, on pourrait aussi prendre une condition entrante de Dirichlet homogène: $\phi_{1}=0$ sur $\Gamma_{i n}$.

A partir de là, on a dans la cavité numéro 1 deux entrées-sorties libres, une en $\Gamma_{0,1}$ et une en $\Gamma_{i n}$. En reprenant les idées de la remarque 1.2, on peut alors jouer sur la fonction $\beta_{1}(t)$ de la formule de Bernoulli, pour créer des dépressions (et "aspirer la membrane"), ou des surpressions (et "gonfler" la membrane). Pour effectuer le bilan énergétique, il suffirait de considérer les estimations avec $g \equiv 0$ (ainsi le terme $\int_{\Gamma_{j}} g \partial_{t} \phi_{1}$ disparaîtrait), mais comme on ne considèrerait plus $\beta_{1} \equiv 0$, on traiterait les termes associés provenant de la formule Bernoulli.

Remarque 1.3. Par ce biais, on peut remplacer le flux d'entrée $g$, qui peut être vu comme une fonction de contrôle en espace et en temps (de $\Gamma_{i n} \times(0, T)$ ), par la variation de $\beta_{1}$, c'est à dire un contrôle qui est une fonction scalaire dépendant uniquement du temps (dans $(0, T)$ ). Par exemple dans le milieu cardiaque, diastole et systole sont réalisées par des variations de pression, et non par un flux de sang en entrée imposé. 


\section{Estimations D'ÉNERGie}

\subsection{Estimations d'énergie des systèmes découplés.}

\subsubsection{Fonctions tests}

Pour obtenir des estimations d'énergie pour chacun des deux systèmes ainsi que pour le système couplé, il faut passer par une étape purement formelle. On intègre par parties les intégrales des équations des systèmes découplés contre des fonctions régulières ad hoc.

Pour la membrane, on multiplie (1) par une fonction-test $b$ définie sur $\gamma \times(0, T)$, et vérifiant les conditions de Dirichlet homogènes $b(x, t)=0$ sur $\partial \gamma \times(0, T)$; on intègre alors sur $\gamma$. Il vient par intégration par parties:

$$
\left.\rho_{s} \int_{\gamma}\left(\partial_{t}^{2} d\right) b+K \int_{\gamma} \partial_{x}\left(\partial_{t} d\right) \partial_{x} b+\sigma \int_{\gamma} \partial_{x} d \partial_{x} b=\int_{\gamma}\left(\mathrm{T}_{\mathrm{FL}} \mathbf{n}_{0}\right)_{y} b \quad \text { pour presque tout } t \in\right] 0, T[.
$$

Pour chacun des deux fluides, on multiplie l'équation de Laplace du système (3) par une fonction-test $\psi_{i}$ définie sur $\Omega_{i}(t)$, appartenant à un espace vectoriel de fonctions suffisamment régulières, et telle que $\psi_{i}=0$ sur $\Gamma_{0, i}$, et on intègre par parties. Il vient:

$$
\begin{array}{ll}
0=\int_{\Omega_{1}(t)} \nabla \phi_{1} \cdot \nabla \psi_{1}-\int_{\Gamma(t)} \partial_{t} d\left(\chi^{-1}, t\right)\left(\mathbf{n}_{t 1}\right)_{y} \psi_{1}-\int_{\Gamma_{i n}} g \psi & \text { pour presque tout } t \in] 0, T[, \\
0=\int_{\Omega_{2}(t)} \nabla \phi_{2} \cdot \nabla \psi_{2}+\int_{\Gamma(t)} \partial_{t} d\left(\chi^{-1}, t\right)\left(\mathbf{n}_{t 1}\right)_{y} \psi_{2} & \text { pour presque tout } t \in] 0, T[.
\end{array}
$$

\subsubsection{Fonctions-tests pour les fluides}

On choisit $\psi_{i}=\partial_{t} \phi_{i}$ comme fonction test. Afin de traiter le premier terme de (5) ou (6), nous rappelons la formule de Reynolds dans un cadre régulier (cf [1] pages 25-26):

Soient le domaine $\Omega(t)$ de normale extérieure unitaire $\mathbf{n}$ et $\mathbf{w}$ la vitesse de la frontière $\partial \Omega(t)$; pour une fonction $\alpha$ donnée, on a:

$$
\frac{d}{d t} \int_{\Omega(t)} \alpha(x, t) d x=\int_{\Omega(t)}\left(\partial_{t} \alpha\right)(x, t) d x+\int_{\partial \Omega(t)} \alpha(y, t) \mathbf{w}(y, t) \cdot \mathbf{n} d y .
$$

Utilisons cette formule avec $\alpha(x, t)=\frac{\left|\nabla \phi_{i}\right|^{2}}{2}$ dans le domaine $\Omega_{i}(t)$, il vient alors:

$$
\begin{aligned}
\int_{\Omega_{i}(t)} \nabla \phi_{i} \cdot \nabla\left(\partial_{t} \phi_{i}\right) d x & =\int_{\Omega_{i}(t)} \partial_{t}\left(\frac{\left|\nabla \phi_{i}\right|^{2}}{2}\right) d x \\
& =\frac{d}{d t} \int_{\Omega_{i}(t)} \frac{\left|\nabla \phi_{i}\right|^{2}}{2} d x+(-1)^{i} \int_{\Gamma(t)} \frac{\left|\nabla \phi_{i}\right|^{2}}{2}\left(\partial_{t} d\left(\chi^{-1}(t, \xi), t\right)\right)\left(\mathbf{n}_{t 1}\right)_{y} d \xi
\end{aligned}
$$

car la vitesse du bord du domaine est nulle sur $\partial \Omega_{i}(t)-\Gamma(t)$, et uniquement transversale sur $\Gamma(t)$ et égale à $\left(0, \partial_{t} d\left(\chi^{-1}, t\right)\right)$.

Remarque 2.1. La vitesse normale de convection du bord du domaine $\Omega_{i}(t)$ à l'interface, $(-1)^{i+1}\left(\partial_{t} d \circ \chi^{-1}\right)\left(\mathbf{n}_{t 1}\right)_{y}$, est précisément égale à $\frac{\partial \phi_{i}}{\partial n}=\mathbf{u}_{i} \cdot \mathbf{n}_{t 1}$ avec $\mathbf{n}_{t 1}=\frac{1}{\sqrt{1+\left(\partial_{x} d\right)^{2}}}\left(\begin{array}{c}-\partial_{x} d \\ 1\end{array}\right)$.

Traitons maintenant à l'aide de la formule de Bernoulli le second terme commun à (5) et (6):

$$
\int_{\Gamma(t)} \partial_{t} d\left(\chi^{-1}, t\right)\left(\mathbf{n}_{t 1}\right)_{y}\left(-\partial_{t} \phi_{i}\right)=\int_{\Gamma(t)} \partial_{t} d\left(\chi^{-1}, t\right)\left(\mathbf{n}_{t 1}\right)_{y}\left(\frac{\left|\nabla \phi_{i}\right|^{2}}{2}+\frac{P_{i}-\beta_{i}}{\rho_{i}}\right)
$$


Ainsi finalement, grâce à (7) et (8), les égalités (5) et (6) s'écrivent respectivement:

$$
\begin{aligned}
& 0=\frac{1}{2} \frac{d}{d t}\left(\int_{\Omega_{1}(t)}\left|\nabla \phi_{1}\right|^{2}\right)-\int_{\Gamma(t)} \partial_{t} d\left(\chi^{-1}, t\right)\left(\mathbf{n}_{t 1}\right)_{y}\left[\frac{\beta_{1}-P_{1}}{\rho_{1}}\right]-\int_{\Gamma_{i n}} g\left(\partial_{t} \phi_{1}\right) \\
& 0=\frac{1}{2} \frac{d}{d t}\left(\int_{\Omega_{2}(t)}\left|\nabla \phi_{2}\right|^{2}\right)+\int_{\Gamma(t)} \partial_{t} d\left(\chi^{-1}, t\right)\left(\mathbf{n}_{t 1}\right)_{y}\left[\frac{\beta_{2}-P_{2}}{\rho_{2}}\right]
\end{aligned}
$$

On a ainsi un bilan d'énergie pour chaque fluide séparément.

\subsubsection{Fonction-test pour la structure}

On prend simplement $b=\partial_{t} d(\cdot, t)$ comme fonction-test, qui est classique et légitime puisque $d(x, t)=0$ au bord $\partial \gamma$ implique $\partial_{t} d(x, t)=0$. On obtient l'estimation d'énergie pour la membrane seule:

$$
\underbrace{\frac{\rho_{s}}{2} \frac{d}{d t} \int_{\gamma}\left|\partial_{t} d\right|^{2}}_{\text {Energie cinétique }}+\underbrace{K \int_{\gamma}\left|\partial_{t}\left(\partial_{x} d\right)\right|^{2}}_{\begin{array}{c}
\text { Dissipation d'énergie } \\
\text { (par visco-élasticité) }
\end{array}}+\underbrace{\frac{\sigma}{2} \frac{d}{d t} \int_{\gamma}\left|\partial_{x} d\right|^{2}}_{\begin{array}{c}
\text { Energie potentielle } \\
\text { (par élongation de la membrane) }
\end{array}}=\int_{\gamma}\left(\mathrm{T}_{\mathrm{FL}} \mathbf{n}_{0}\right)_{y}\left(\partial_{t} d\right)
$$

\subsection{Estimation d'énergie du système couplé global à deux cavités}

On somme les bilans d'énergies de la membrane (11), du premier fluide (9), et du second fluide (10):

$$
\begin{aligned}
& \frac{\rho_{s}}{2} \frac{d}{d t} \int_{\gamma}\left|\partial_{t} d\right|^{2}+K \int_{\gamma}\left|\partial_{t}\left(\partial_{x} d\right)\right|^{2}+\frac{\sigma}{2} \frac{d}{d t} \int_{\gamma}\left|\partial_{x} d\right|^{2} \\
& \quad+\frac{1}{2} \rho_{1} \frac{d}{d t}\left(\int_{\Omega_{1}(t)}\left|\nabla \phi_{1}\right|^{2}\right)+\frac{1}{2} \rho_{2} \frac{d}{d t}\left(\int_{\Omega_{2}(t)}\left|\nabla \phi_{2}\right|^{2}\right) \\
& \quad=\rho_{1} \int_{\Gamma_{i n}} g\left(\partial_{t} \phi_{1}\right)+\int_{\gamma}\left[\left(\mathrm{T}_{\mathrm{FL}} \mathbf{n}_{0}\right)_{y}+\left(\left(\left[\beta_{1}-P_{1}\right]-\left[\beta_{2}-P_{2}\right]\right) \circ \chi\right)\left(\mathbf{n}_{0}\right)_{y}\right]\left(\partial_{t} d\right)
\end{aligned}
$$

en ayant réécrit en coordonnées lagrangiennes sur $\gamma$ l'intégrale de bord sur l'interface $\Gamma(t)$. En tenant compte de la condition de couplage pour l'égalité des tenseurs des contraintes sur l'interface, cette dernière intégrale disparaît.

A partir de là, on définit l'énergie totale $E_{S y s t}$ du système pour tout $t \in[0, T]$ :

$$
E_{S y s t}(t)=\underbrace{\frac{1}{2} \sum_{i=1,2} \rho_{i}\left\|\nabla \phi_{i}(t)\right\|_{L^{2}\left(\Omega_{i}(t)\right)}^{2}}_{\begin{array}{c}
\text { Energies cinétiques } \\
\text { des fluides 1 et } 2
\end{array}}+\underbrace{\frac{1}{2} \rho_{s}\left\|\partial_{t} d\right\|_{L^{2}(\gamma)}^{2}}_{\begin{array}{c}
\text { Energie cinétique } \\
\text { de la membrane }
\end{array}}+\underbrace{\frac{1}{2} \sigma\left\|\partial_{x} d\right\|_{L^{2}(\gamma)}^{2}}_{\begin{array}{c}
\text { Energie potentielle } \\
\text { membranaire } \\
\text { élastique }
\end{array}}
$$

D'après ce qui précède, celle-ci vérifie l'égalité suivante:

$$
\forall \tau \in[0, T], \quad \underbrace{\frac{d}{d t} E_{S y s t}(\tau)}_{\text {Variation Energie Totale }}=\underbrace{\rho_{1} \int_{\Gamma_{\text {in }}} g\left(\partial_{t} \phi_{1}\right)}_{\text {Apport d'énergie }}-\underbrace{K \int_{\gamma}\left|\partial_{x}\left(\partial_{t} d\right)\right|^{2}}_{\text {Dissipation }} .
$$

Remarque 2.2. Pour un système isolé, c'est à dire sans entrée de fluide $(g \equiv 0)$, l'énergie totale $E_{\text {Syst }}(t)$ du système à chaque instant t est décroissante (strictement par exemple, dès que la vitesse initiale est non-nulle). 
Notons par ailleurs que le terme $K \int_{\gamma}\left|\partial_{x}\left(\partial_{t} d\right)\right|^{2}$ fait intervenir la raideur de la membrane: plus $K$ est élevé, plus l'énergie se dissipe rapidement.

Si on intègre cette variation d'énergie totale entre $\left[t_{0}, t_{1}\right] \subset[0, T]$, on obtient:

$$
E_{\text {Syst }}\left(t_{1}\right)=E_{\text {Syst }}\left(t_{0}\right)+\rho_{1} \int_{\Gamma_{\text {in }}} \int_{t_{0}}^{t_{1}} g \partial_{t} \phi_{1}-K \int_{t_{0}}^{t_{1}} \int_{\gamma}\left|\partial_{x}\left(\partial_{t} d\right)\right|^{2}
$$

On peut exprimer le terme lié à l'injection de fluide de différentes façons:

1) soit par intégration par parties en temps:

$$
\int_{t_{0}}^{t_{1}} g\left(\partial_{t} \phi_{1}\right)=-\int_{t_{0}}^{t_{1}}\left(\partial_{t} g\right) \phi_{1}+\left(g\left(t_{1}\right) \phi_{1}\left(t_{1}\right)-g\left(t_{0}\right) \phi_{1}\left(t_{0}\right)\right)
$$

et en supposant qu'aux temps $t_{0}$ et $t_{1}$ il n'y a pas d'injection de fluide, $g\left(t_{0}\right)=g\left(t_{1}\right)=0$ sur $\Gamma_{i n}$, c'est alors la variation $\partial_{t} g$ du flux en entrée qui est mise en valeur.

2) soit en utilisant la formule de Bernoulli sur $\Gamma_{i n}$ :

$$
\int_{\Gamma_{i n}} \int_{t_{0}}^{t_{1}} g \partial_{t} \phi_{1}=\int_{\Gamma_{\text {in }}} \int_{t_{0}}^{t_{1}} g\left(-\frac{\left|\nabla \phi_{1}\right|^{2}}{2}+\frac{\beta_{1}-P_{1}}{\rho_{1}}\right)
$$

si on fixe la fonction $\beta_{1}(t)$ telle que $\left(\beta_{1}-P_{1}\right)<0$ (par exemple avec une pression en aval plus faible dans le réservoir $\mathcal{R}_{1}$ par lequel s'échappe le fluide), puisque le flux $g$ est lui aussi négatif (pour une entrée de fluide selon la normale extérieure), on voit que ce terme est positif, et apporte donc de l'énergie au système global. En effet, $\int_{\Gamma_{i n}} \int_{t_{0}}^{t_{1}} g\left(\frac{\beta_{1}-P_{1}}{\rho_{1}}\right)$ s'assimile à un travail de la pression entre l'entrée et la sortie de la cavité, tandis que $\int_{\Gamma_{i n}} \int_{t_{0}}^{t_{1}} g\left(-\frac{\left|\nabla \phi_{1}\right|^{2}}{2}\right)$ est comparable au terme $\int_{t_{0}}^{t_{1}} \int_{\Gamma_{i n}} \mathbf{u}^{2}(\mathbf{u} \cdot \mathbf{n})$, issu des estimations d'énergie des équations de Navier-Stokes dans un cadre similaire, et qui s'interprète comme un flux d'énergie cinétique.

Finalement on obtient un encadrement de l'énergie totale du système à l'instant $t_{1}$ :

$$
E_{S y s t}\left(t_{0}\right)-K \int_{t_{0}}^{t 1} \int_{\gamma}\left|\partial_{x}\left(\partial_{t} d\right)\right|^{2} \leq E_{S y s t}\left(t_{1}\right) \leq E_{S y s t}\left(t_{0}\right)+\rho_{1} \int_{\Gamma_{\text {in }}} \int_{t_{0}}^{t 1} g\left(\partial_{t} \phi_{1}\right) .
$$

On constate ainsi que si $g$ est nulle sur l'intervalle de temps $\left[t_{0}, t_{1}\right]$, c'est à dire si le système est isolé, alors l'énergie est bornée uniformément sur $\left[t_{0}, t_{1}\right]$, ce que l'on peut exploiter pour montrer l'existence d'une solution au problème théorique.

Remarque 2.3. On possède une information supplémentaire sur g: le flux est conservatif. En effet, le fluide étant incompressible, on intègre les relations d'incompressibilité sur les vitesses div $\mathbf{u}_{1}=0$ sur $\Omega_{1}(t)$ et div $\mathbf{u}_{2}=$ 0 sur $\Omega_{2}(t)$ que l'on somme après intégrations par parties et ainsi à chaque instant:

$$
\int_{\Gamma_{i n}}(-g)=\int_{\Gamma_{0,1}} \frac{\partial \phi_{1}}{\partial n}+\int_{\Gamma_{0,2}} \frac{\partial \phi_{2}}{\partial n}
$$

Tout le fluide entrant par $\Gamma_{\text {in }}$ à chaque instant est égal à la somme des flux entrant et sortant par $\Gamma_{0,1}$ et $\Gamma_{0,2}$, et il n'y a pas de modification du volume global des fluides à l'intérieur de la cavité. 


\section{Algorithme. Résultats numériques}

\subsection{Discrétisation des équations}

\subsubsection{Synthèse modale pour la structure}

Le déplacement transversal de la membrane est régi par le système (1). Pour la discrétisation en espace, on utilise les $N$ premiers vecteurs propres $\psi_{j}$ de l'opérateur stationnaire de l'élasticité linéaire associé à notre problème, avec des conditions au bord de Dirichlet homogènes:

$$
\left\{\begin{array}{cll}
-\partial_{x}^{2} \psi_{j} & =\lambda_{j} \psi_{j} & \text { dans } \gamma \\
\psi_{j} & =0 & \text { sur } \partial \gamma
\end{array}\right.
$$

Ce qui, dans notre cas 1d, est facilement explicité (en notant $|\gamma|$ la longueur de $\gamma$ ):

$$
\left(\lambda_{j}, \psi_{j}(\xi)\right)=\left(\left(\frac{j \pi}{|\gamma|}\right)^{2}, \sin \left(\frac{j \pi \xi}{|\gamma|}\right)\right) .
$$

En fait, numériquement, quelques modes propres suffisent ( $N=5$ par exemple) pour prendre en compte les basses fréquences et obtenir une simulation raisonnable de la membrane.

Pour la discrétisation en temps, on utilise un schéma aux différences finies stable de Newmark d'ordre 2.

\subsubsection{Formulation ALE et éléments finis pour chaque fluide}

La procédure principale est la même pour chacun des deux fluides. Seule la prise en compte des conditions aux bords change (par exemple à l'interface). De façon synthétique, nous avons donc à résoudre un laplacien avec différentes conditions aux limites sur un domaine $\Omega_{n} \stackrel{\text { déf }}{=} \Omega\left(t_{n}\right)$ qui évolue en temps. Pour un temps $t_{n}$ fixé, nous approchons le laplacien par une méthode d'éléments finis $\left(P_{1}\right)$ sur une triangulation du domaine $\Omega_{n}$ donnant un maillage structuré.

Comme le domaine fluide est mobile, nous utilisons une formulation ALE (voir par exemple [4]) simplifiée. En effet, comme aucun opérateur en temps n'agit sur le potentiel dans le système (3), on n'a pas besoin de réécrire le système sur un domaine spatial différent, ni de faire intervenir la convection du domaine dans le système discrétisé. Il suffit de résoudre un laplacien à chaque instant dans un domaine fixé $\left(\Omega_{n}\right)$, domaine qui lui est convecté dans une autre étape du calcul.

Nous construisons la matrice éléments finis $M$ du laplacien à partir du maillage déformé. La matrice obtenue est symétrique; elle est définie pourvu qu'il y ait au moins un sommet parmi les points du maillage qui rende compte d'une condition de type Dirichlet. Par ailleurs, les conditions de Dirichlet au bord sont imposées par une méthode de pénalisation des coefficients ad hoc. Pour inverser la matrice $M$, nous employons une factorisation de Cholesky (en utilisant la structure de matrice-bande pour obtenir des gains de stockage).

Une fois obtenu le potentiel $\phi\left(t_{n}, \cdot\right)$, nous retrouvons la vitesse $\mathbf{u}\left(t_{n}, \cdot\right)$ de façon variationnelle . Pour cela, on note $w_{i}$ la fonction chapeau scalaire (élément fini $P_{1}$ ici) associée au $i$-ième sommet du maillage; et pour chaque couple $(i, j)$ de sommets, on pose $\mathbf{W}_{i j}=w_{i} \mathbf{e}_{x}+w_{j} \mathbf{e}_{y}$, et on note $\mathcal{W}$ la base formée par l'ensemble de ces fonctions vectorielles. On écrit alors:

$$
\int_{\Omega_{n}} \mathbf{u} \cdot \mathbf{W}=\int_{\Omega_{n}} \nabla \phi \cdot \mathbf{W}
$$

avec $\mathbf{W}$ un élément de $\operatorname{Vect}(\mathcal{W})$ (l'espace vectoriel engendré par $\mathcal{W})$. En décomposant u sur la base $\mathcal{W}$, on obtient pour le membre de gauche la matrice identité sur le maillage déformé. Tandis que pour le membre de droite, en décomposant $\nabla \phi$, on aura à calculer la matrice (en séparant les coordonnées en $x$ et $y$ ):

$$
\left(\begin{array}{c|c}
\int_{\Omega_{n}}\left(\nabla \mathbf{w}_{i}\right) \mathbf{w}_{k} & 0 \\
\hline 0 & \int_{\Omega_{n}}\left(\nabla \mathbf{w}_{j}\right) \mathbf{w}_{l}
\end{array}\right) .
$$



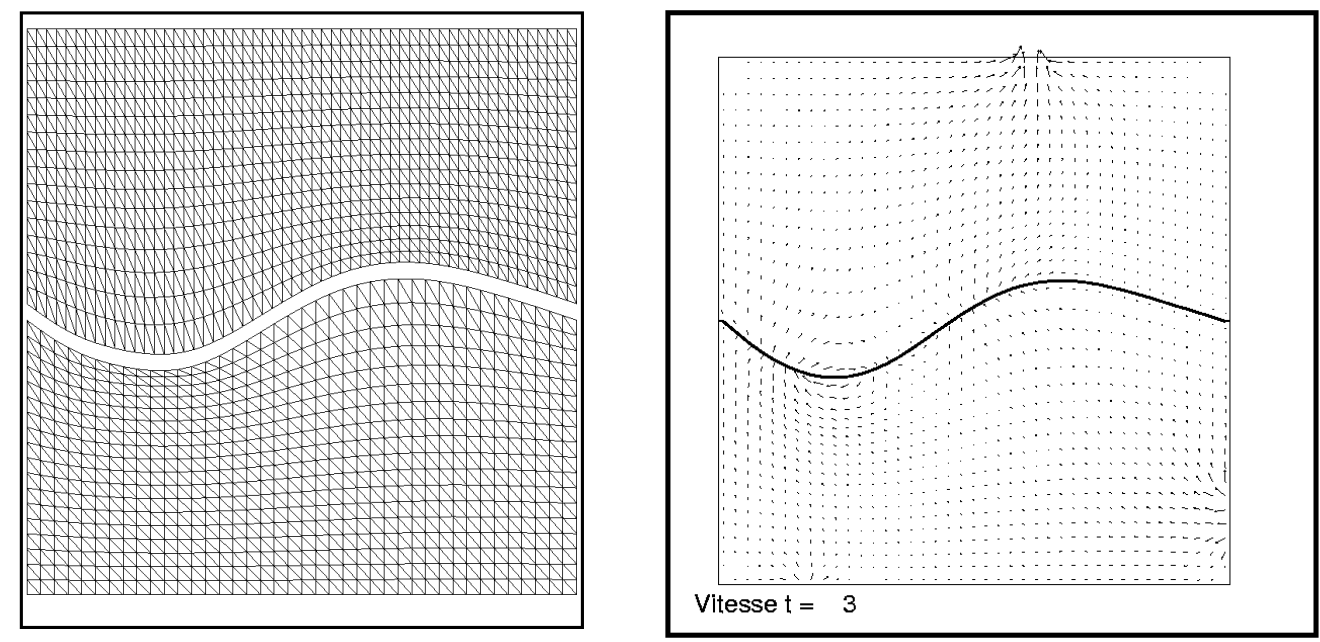

FIgURE 2. Maillage et vitesses au temps $\mathrm{t}=3$.

Une fois retrouvée la vitesse $\mathbf{u}\left(t_{n}, \cdot\right)$, on calcule la pression $P\left(t_{n}, \cdot\right)$ à partir de l'identité de Bernoulli, par l'équation scalaire discrétisée suivante :

$$
\frac{\phi^{n+1}-\phi^{n}}{\Delta t}+\frac{P^{n+1}}{\rho_{i}}+\frac{\left(\mathbf{u}^{n+1}\right)^{2}}{2}=C\left(t_{n}\right) \quad \text { dans } \Omega_{n}
$$

où l'on peut faire varier $C\left(t_{n}\right)$ pour créer des dépressions ( $c f$ remarques 1.2 et 1.3 ) .

\subsubsection{Algorithme de couplage}

Nous adoptons un algorithme de couplage dit "faible" au sens où le problème des fluides et le problème de la structure sont résolus tour à tour à chaque itération en temps (et non simultanément).

\section{Algorithme de résolution du système couplé}

On note $d^{n}$ le déplacement au temps $t_{n} ; \mathcal{M}_{i}^{n}$ le maillage $\mathcal{M}_{i}, \phi_{i}^{n}$ le potentiel $\phi_{i}, P_{i}^{n}$ la pression $P_{i}$ de la cavité $i \in\{1,2\}$ au temps $t_{n}$. Voici l'algorithme qui nous avons adopté:

1. $d^{n-1}, d^{n}, \mathcal{M}_{i}^{n}, \phi_{i}^{n}, P_{i}^{n}$ donnés au temps $t_{n}$.

2. Résolution de la structure: $\longrightarrow d^{n+1}$.

3. $\quad$ Calcul de la vitesse de déplacement de l'interface.

4. Mise à jour des maillages (par formulation ALE): $\longrightarrow \mathcal{M}_{i}^{n+1}$.

5. Calculs des matrices de rigidité pour les laplaciens.

6. Résolution des potentiels avec les conditions aux bords: $\longrightarrow \phi_{i}^{n+1}$.

7. Calcul des pressions: $\longrightarrow P_{i}^{n+1}$.

8. Calcul des contraintes fluides au temps $t_{n+1}$.

9. $n:=n+1$, itération suivante (aller en (1)).

\subsection{Résultats numériques}

Nous considérons maintenant les figures $2,3,4,5$ qui se rapportent au même cas test, avec en entrée de fluide une fonction de profil parabolique en espace et sinusoïdal en temps et qui s'annule pour $t \geq 150$. Les déplacements sont de l'ordre du vingtième des longueurs caractéristiques de la cavité. 

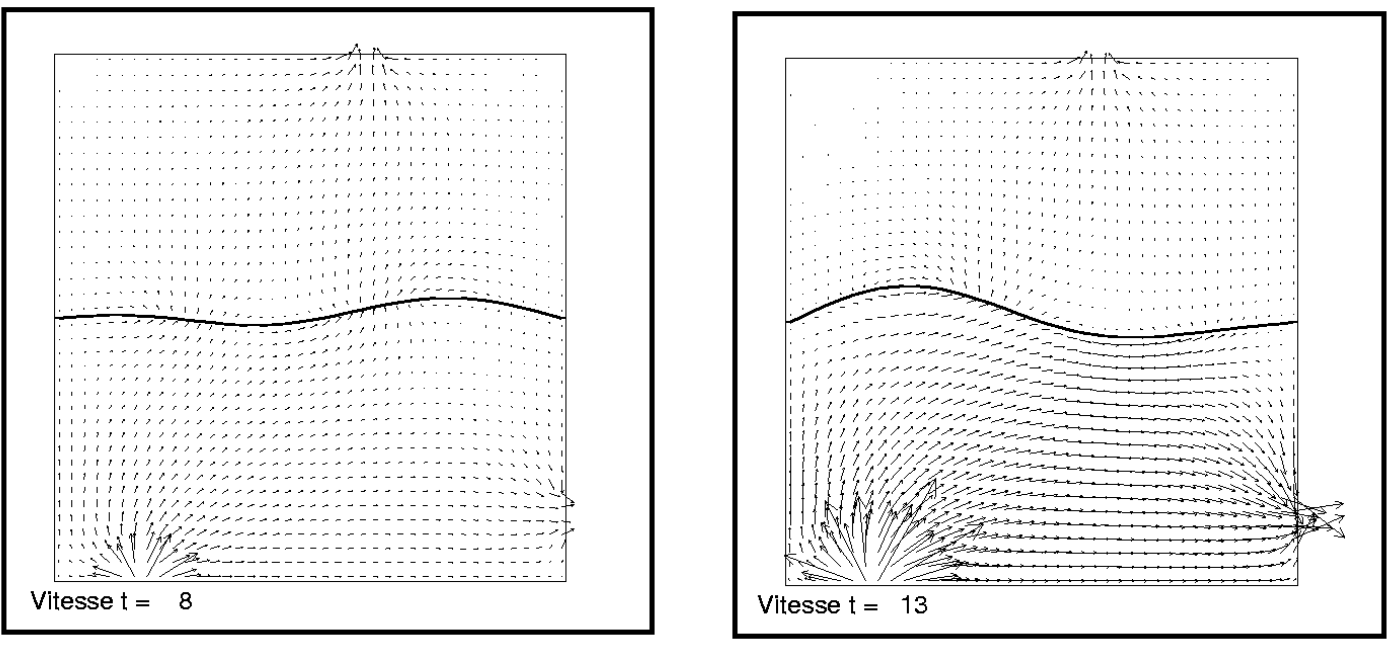

FigURE 3. Vitesses aux temps $\mathrm{t}=8$ et $\mathrm{t}=13$.
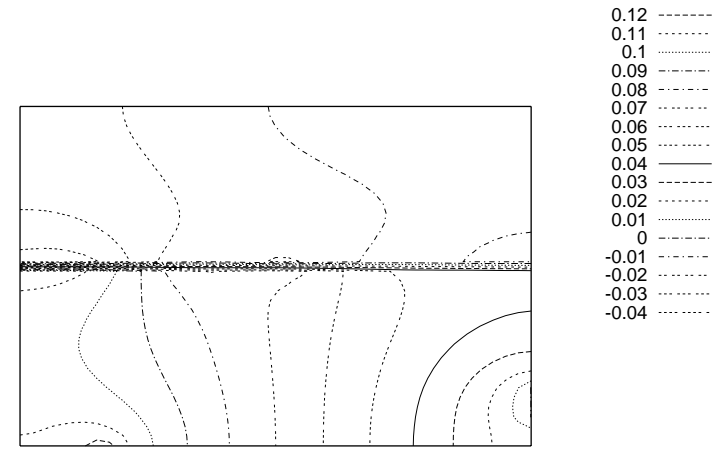

FiguRE 4. Isovaleurs de la pression au temps $\mathrm{t}=8$.

Les figures 2 et 3 présentent des photographies du champ des vitesses à trois instants différents. La première ( $c f$ Figure 2), au temps $t=3$, présente une injection de fluide dans la cavité 1 par l'ouverture libre $\Gamma_{0,1}$ due à la réaction de la membrane élastique par rapport à son état initial (déplacement non nul, mais vitesse initiale nulle); le fluide 1 commence à rentrer par $\Gamma_{i n}$ de façon forcée; le fluide 2 sort librement par $\Gamma_{0,2}$. Au temps $t=8$ ( $c f$ Figure 3.a), l'injection est plus importante, si bien qu'elle implique la sortie du fluide par $\Gamma_{0,1}$; par ailleurs, la membrane répond à cette différence de pression et continue aussi de réagir par rapport à son écart initial à sa position d'équilibre. Au temps $t=13$ ( $c f$ Figure 3.b), le fluide 1 est toujours dans sa phase d'injection ( ar $\Gamma_{i n}$ ), et la membrane subit des efforts plus importants; la vitesse dans le fluide 1 continue d'augmenter (en particulier en sortie $\Gamma_{0,1}$ ); celle du fluide 2 reste sensiblement la même, car seules les vitesses normales sont "échangées" à l'interface $\Gamma(t)$. 


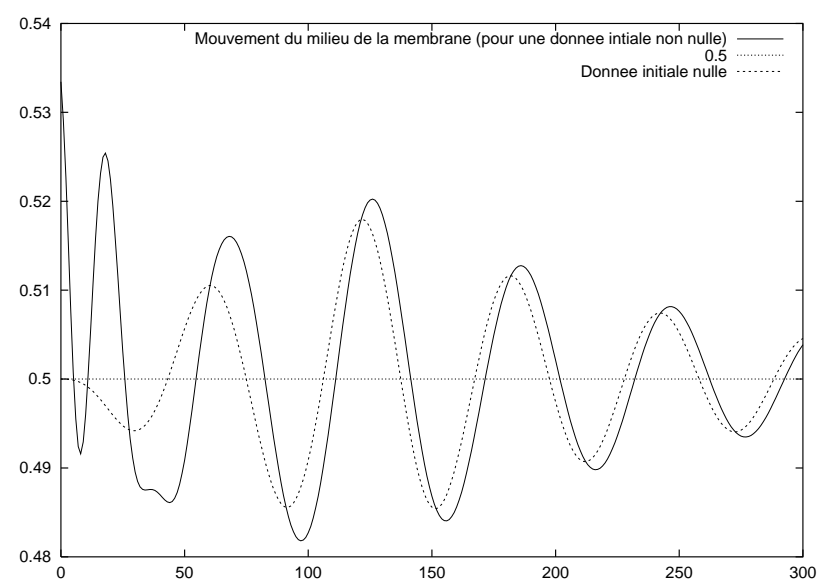

Figure 5. Evolution de la seconde composante du point milieu de la membrane avec et sans déplacement initial (avec la longueur d'un côté de la cavité égal à 1).

La figure 4 représente les isovaleurs des pressions dans les deux cavités fluides à l'instant $t=8$, coïncidant avec la figure 3.a des vitesses au même instant. Enfin, la figure 5 représente l'évolution en temps de l'écart (en ordonnée) du point milieu de la membrane par rapport à son niveau d'équilibre dans deux cas: avec ou sans déplacement initial. En particulier, la déformation suit bien la forme sinusoïdale qu'on impose en entrée pour le flux. Par ailleurs, le cas où la position initiale de la membrane n'est pas nulle "recolle" à celui où la position initiale est nulle après un certain temps à un décalage de phase près. Juste après l'arrêt de l'injection de fluide $(t \geq 150)$, on observe une décroissance de la position de la membrane et asymptotiquement un retour à la position d'équilibre (qui est ici la position plane dans notre cas où l'on n'a pas créé de différence de pression entre les deux cavités et où l'on a pris les fonctions $\left.\beta_{i}(t) \equiv 0\right)$.

Paramètres numériques: les densités des fluides: $\rho_{1}=\rho_{2}=1$; le triplet de constantes caractéristique de la membrane $\left(\rho_{s}, \sigma, K\right)=(10,1.1,0.15)$; le pas de temps $\Delta t=0.1$; le nombre de modes propres $N_{\text {mod }}=10 ;$ la longueur et la largeur de la cavité $L=1$; les fonctions $\beta_{i} \equiv 0$; les nombres de points pour les maillages des cavités fluides (qui peuvent être différents):779 et 697; l'amplitude de la fonction d'entrée est environ 2.

\section{REFERENCES}

[1] Georges DUVAUT, Mécaniques des Milieux continus, Collection Mathématiques Appliquées pour la maîtrise, Masson, 1990.

[2] Céline GRANDMONT, Analyse mathématique et numérique de quelques problèmes d'interaction fluide-structure, Thèse de doctorat, Université Pierre et Marie Curie, 1998.

[3] Patrick HUERRE, Mécaniques des Milieux Continus, Tome 4, Mécanique des fluides, École Polytechnique, 1994.

[4] Bertrand MAURY, Characteristics ALE Method for the Unsteady 3D Navier-Stokes Equations with a Free Surface, International Journal of Computational Fluid Dynamics 6, pages 175-188, 1996. 